\title{
Ionospheric Effects on High Gain Antenna GNSS Measurements - TEC Estimation and Correction
}

\author{
Steffen Thoelert, German Aerospace Center (DLR), Germany \\ Ulrich Hörmann, University of Potsdam, Germany \\ Felix Antreich, Department of Teleinformatics Engineering, Federal University of Ceará (UFC), Fortaleza, Brazil \\ Michael Meurer, German Aerospace Center (DLR) \& RWTH Aachen University, Germany
}

\section{BIOGRAPHY (IES)}

Steffen Thoelert received his diploma degree in electrical engineering from the University of Magdeburg in 2002. In May 2006 he joined the Institute of Communications and Navigation at the German Aerospace Centre (DLR), Oberpfaffenhofen. His current research activities include signal quality monitoring, satellite payload characterization, system calibration, GNSS compatibility and interference aspects.

Ulrich Hörmann studied physics in Augsburg (Germany) and Karlstad (Sweden). He received his Ph.D. in physics from the University of Augsburg in 2015 and joined the Institute of Communications and Navigation at the German Aerospace Center (DLR) with his focus on GNSS signal monitoring. Recently, he has moved to the Institute of Physics and Astronomy at the University of Potsdam.

Felix Antreich received the Diploma degree in electrical engineering from the Munich University of Technology (TUM), Munich, Germany, in 2003. In 2011 he also received the Doktor-Ingenieur (Ph.D.) degree from the TUM. Since September 2016 he is a visiting professor in the Department of Teleinformatics Engineering (DETI) at the Federal University of Ceará (UFC) in Fortaleza, Brazil.

Michael Meurer received the diploma in Electrical Engineering and the Ph.D. degree from the University of Kaiserslautern, Germany. Since 2006 he is the director of the Department of Navigation of the German Aerospace Center (DLR). In addition, he is a professor of electrical engineering and director of the Chair of Navigation at the RWTH Aachen University. His current research interests include GNSS signals, GNSS receivers, and navigation for safety-critical applications.

\begin{abstract}
The ionospheric delay of global navigation satellite systems (GNSS) signals typically is compensated by adding a single correction value to the pseudorange measurement of a GNSS receiver. Yet, this neglects the dispersive nature of the ionosphere. In this context we analyze the ionospheric signal distortion beyond a constant delay. These effects become increasingly significant with the signal bandwidth and hence more important for new broadband navigation signals. Using measurements of the Galileo E5 signal, captured with a high gain antenna, we verify that the expected influence can indeed be observed and compensated. A new method to estimate the total electron content (TEC) from a single frequency high gain antenna measurement of a broadband GNSS signal is proposed and described in detail. The received signal is de facto unaffected by multi-path and interference because of the narrow aperture angle of the used antenna which should reduce the error source of the result in general. We would like to point out that such measurements are independent of code correlation, like in standard receiver applications. It is therefore also usable without knowledge of the signal coding. Results of the TEC estimation process are shown and discussed comparing to common TEC products like TEC maps and dual frequency receiver estimates.
\end{abstract}

\section{INTRODUCTION}

The ionosphere is a part of the earth's atmosphere which starts at about $50 \mathrm{~km}$ and extends to roughly $1000 \mathrm{~km}$ height (Zolesi et al. 2014). In these layers, solar radiation ionizes a fraction of the present molecules and hence generates a plasma (Kelley 2009). For radio signals traveling from space to earth or vice versa, this conductive layer represents a dispersive medium. In a global navigation satellite systems (GNSS) receiver usually, corrections for the phase advance and the group delay introduced by the propagation of the satellite signal through the ionosphere are corrected with a single coefficient added to the code phase 
and carrier phase pseudorange measurement. However, this neglects the dispersive nature of the ionosphere, especially for broadband signals (Gao et al. 2007, Henkel et al. 2009). For some applications like precise positioning (Henkel et al. 2009) or GNSS signal verification (Thoelert et al. 2013) the correction of the introduced group delay and phase advance by one single coefficient in the carrier phase and code phase pseudoranges does not provide sufficient accuracy in correcting these ionospheric effects. In case of signal verification, there is a great need for broadband ionospheric corrections with high accuracy in order to assess the characteristics of the payload and to perform signal quality monitoring (SQM). In this case, it is also desired to derive ionospheric corrections immediately during the measurements with a high gain antenna and usually only observing one GNSS frequency band. Usually, total electron (TEC) maps derived based on a network of GNSS receivers are not available in real-time and are only available with a certain delay. Thus, TEC maps are only useful in post-processing the high gain measurement data, but not for real-time ionospheric corrections.

In this work, we explore how the dispersive nature of the ionosphere is affecting broadband signals, in particular, how the socalled signal constellation diagram is affected. Furthermore, we will present a novel method to estimate slant TEC based on single frequency high gain antenna measurements and we show that based on these estimates the dispersive nature of the ionosphere can be taken into account for broadband GNSS signals ionospheric corrections. Such analysis, estimation of slant TEC, and ionospheric corrections for broadband signals are especially interesting for SQM and signal verification using high gain antennas.

In the section below we define the signal model after propagating through the ionosphere and after downconversion to baseband. Afterwards, we will derive an estimator for single frequency slant TEC estimation based on binned-data (signal constellation diagram). Finally, we will assess the dispersive effect of the ionosphere in broadband signals and we will analyze the performance of our new approach to estimate slant TEC based on high gain antenna measurements.

\section{SIGNAL MODEL}

A digitally modulated bandpass GNSS signal transmitted by a satellite can be given by

$$
\tilde{y}(t)=\sqrt{P_{t}}\left(y_{I}(t) \cos \left(2 \pi f_{c} t\right)-y_{Q}(t) \sin \left(2 \pi f_{c} t\right)\right.
$$

where $\mathrm{y}_{\mathrm{I}}(\mathrm{t})$ is the so-called inphase component and $\mathrm{y}_{\mathrm{Q}}(\mathrm{t})$ quadrature component. $\mathrm{P}_{\mathrm{t}}$ is the transmit power and $\mathrm{f}_{\mathrm{c}}$ is the carrier frequency of the signal. The Fourier transform of $\tilde{y}(t)$ is denoted by $\mathcal{F}\{\tilde{y}(t)\}=\widetilde{Y}(f)$ and the respective inverse Fourier transform is given by $\mathcal{F}^{-1}\{\widetilde{\mathrm{Y}}(\mathrm{f})\}=\tilde{\mathrm{y}}(\mathrm{t})$. Propagation of the signal through the ionosphere can be approximated by the transfer function

$$
H(f ; T E C)=e^{j 2 \pi f \Delta T(f ; T E C)}
$$

with $\triangle T(f ; T E C)$ being the group delay introduced by the propagation of the signal through the ionosphere. Please note, that the inverse Fourier transform of $H(f ; T E C)$ cannot be derived as the integral $\int_{-\infty}^{\infty} H(f ; T E C) e^{j 2 \pi f t} d f$ does not converge. However, the inverse Fourier transform of $\tilde{Y}(f) H(f ; T E C)$ can be derived in case $\tilde{y}(t)$ is bandlimited to the single-sided bandwidth $B$ and $f_{c}>B$. In the following, besides the ionosphere, we only consider free space path-loss $L$, and no other effects caused by the troposphere, multipath, etc.. Following (Hoibinger and Jakowski 2017) the troposphere can be considered to be a nondispersive medium and consequently troposphere delay effects are identical for group and phase delay observations. Thus, the troposphere does not affect estimates of the slant TEC based on the dispersion influencing GNSS signals. Multipath can be neglected, as for the beamwidth of the high gain antenna pattern is very small and the sidelobe to mainlobe gain ratio is large.

Thus, the received signal after propagating from the satellite to the receiver on earth can be given by

$$
\sqrt{L G_{r} G_{t}} \tilde{Y}(f) H(f ; T E C)=\frac{\sqrt{P_{r}}}{2} Y\left(f-f_{c}\right) H(f ; T E C)+\frac{\sqrt{P_{r}}}{2} Y^{*}\left(f+f_{c}\right) H(f ; T E C)
$$

where $G_{r}$ is the gain of the receive antenna, $G_{t}$ is the gain of the transmit antenna, $P_{r}=P_{t} L G_{r} G_{t}$ is the received power, $Y(f)=Y_{I}(f)+j Y_{Q}(f), \mathcal{F}\left\{y_{I}(t)\right\}=Y_{I}(f)$, and $\mathcal{F}\left\{y_{Q}(t)\right\}=Y_{Q}(f)$. Performing down-conversion of the signal by multiplying the passband signal with either $2 \cos \left(2 \pi f_{c} t\right)$ or $-2 \sin \left(2 \pi f_{c} t\right)$ and subsequent low-pass filtering with

$$
\mathcal{F}\left\{h_{L}(t)\right\}=H_{L}(f)=\left\{\begin{array}{l}
1,|f| \leq B \\
0,|f|>B
\end{array}\right.
$$


as depicted in Figure 1 we get

$$
\mathcal{F}\left\{s_{I}(t ; \theta)\right\}=\frac{\sqrt{P_{r}}}{2} Y^{*}(f) H\left(f-f_{c} ; T E C\right)+\frac{\sqrt{P_{r}}}{2} Y(f) H\left(f+f_{c} ; T E C\right)
$$

and

$$
\mathcal{F}\left\{s_{Q}(t ; \boldsymbol{\theta})\right\}=\frac{j \sqrt{P_{r}}}{2} Y^{*}(f) H\left(f-f_{c} ; T E C\right)-\frac{j \sqrt{P_{r}}}{2} Y(f) H\left(f+f_{c} ; T E C\right)
$$

where

$$
\boldsymbol{\theta}=\left[T E C, P_{r}\right]^{T}
$$
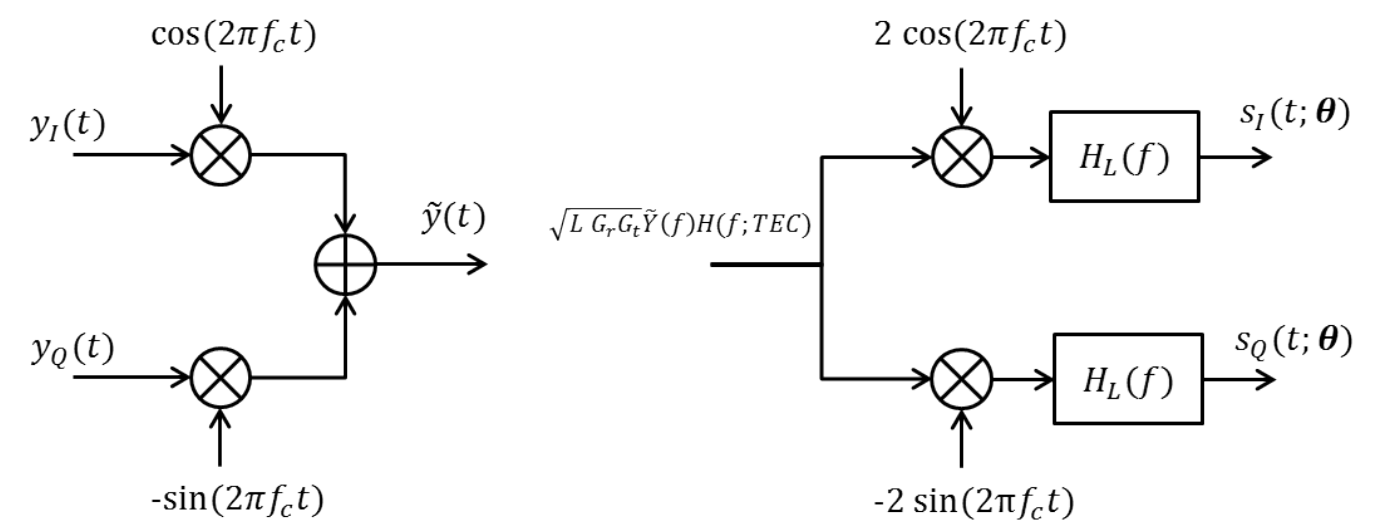

Fig. 1 Baseband receive signal model.

The complex envelope of received signal can be given by

$$
\mathcal{F}\{s(t ; \boldsymbol{\theta})\}=\mathcal{F}\left\{s_{I}(t ; \boldsymbol{\theta})+j s_{Q}(t ; \boldsymbol{\theta})\right\}=\sqrt{P_{r}} Y(f) H\left(f-f_{c} ; T E C\right)=\sqrt{P_{r}}\left(Y_{I}(f)+j Y_{Q}(f)\right) H\left(f-f_{c} ; T E C\right)
$$

\section{MAXIMUM LIKELIHOOD ESTIMATION OF TEC AND RECEIVED POWER BASED ON BINNED DATA}

We consider $N$ observations of a random vector $\boldsymbol{x}$ which has a multivariate Gaussian probability density function (pdf) parameterized by the parameter vector $\boldsymbol{\theta}$, denoted by $p_{\boldsymbol{x}}(\mathbf{x}[n] ; \boldsymbol{\theta})$. The realization of the random variable $\boldsymbol{x}$ at time instant $n$ is given by $\mathbf{x}[n]$ and $n=1, \ldots, N$. The pdf is given by

$$
p_{\boldsymbol{x}}(\boldsymbol{x}[n] ; \boldsymbol{\theta})=\frac{1}{\sqrt{2 \pi \sigma_{n}^{2}}} \exp \left[-\frac{\|\mathrm{x}[n]-s[n ; \theta]\|_{2}^{2}}{2 \sigma_{n}^{2}}\right]
$$

where the discrete signal model with $t=n T_{s}$ and $T_{s}=1 / 2 B$ can be given by

$$
\mathbf{x}[n]=\left[\begin{array}{l}
x_{I}[n] \\
x_{Q}[n]
\end{array}\right]=\mathbf{s}[n ; \theta]+\mathbf{n}[n]=\left[\begin{array}{l}
s_{I}[n ; \theta] \\
s_{Q}[n ; \theta]
\end{array}\right]+\left[\begin{array}{l}
n_{I}[n] \\
n_{Q}[n]
\end{array}\right] \in \mathbb{R}^{2 \times 1} .
$$

Here, $x_{I}[n]$ and $x_{Q}[n]$ are the inphase and quadrature components of the received signal and $n_{I}[n]$ and $n_{Q}[n]$ are the inphase and quadrature components of the additive noise with variance $\sigma_{n}^{2}$. We assume that the noise is white Gaussian and proper. The likelihood is given by

$$
L(\mathbf{x}[n] ; \boldsymbol{\theta})=p_{\boldsymbol{x}}(\mathbf{x}[n] ; \boldsymbol{\theta}) .
$$

For large data samples $(N \rightarrow \infty)$, the log-likelihood function becomes difficult to compute since one has to sum $\log (L(\mathbf{x}[n] ; \boldsymbol{\theta}))$ for all observations $N$. In such cases instead of recording all the observations we can build a histogram with a 
number of entries $\mathbf{b}=\left[b_{1}, \ldots, b_{M}\right]^{T} \in \mathbb{N}_{0}^{M \times 1}$ in $M$ bins with $m=1, \ldots, M$ as outlined in G. Cowan (1998). The expectation $\epsilon(\theta)=\left[\epsilon_{1}(\theta), \ldots, \epsilon_{M}(\theta)\right]^{T} \in \mathbb{N}_{0}^{M \times 1}$ of the number of entries $b_{m}$ can be given by

$$
\epsilon_{m}(\boldsymbol{\theta})=\sum_{\mathbf{x}_{m}^{\min } \leq \mathbf{x}[n]<\mathbf{x}_{m}^{\max }} L(\mathbf{x}[n] ; \boldsymbol{\theta})=N p_{m}(\boldsymbol{\theta})
$$

where $\mathbf{x}_{m}^{\min }$ and $\mathbf{x}_{m}^{\max }$ are the bin limits of the histogram and $p_{m}(\boldsymbol{\theta})$ is the probability of an entry in the $m$-th bin of the histogram. We define vector inequality (as used above in (12)) of two vectors $\mathbf{o}=\left[o_{1}, \ldots, o_{N}\right]^{T}$ and $\mathbf{p}=\left[p_{1}, \ldots, p_{N}\right]^{T}$ with $\mathbf{o}, \mathbf{p} \in \mathbb{R}^{N \times 1}$ as

$$
\begin{aligned}
& \mathbf{o} \leq \mathbf{p} \text { if } \forall_{i=1}^{N} o_{i} \leq p_{i} \\
& \mathbf{o}<\mathbf{p} \text { if } \forall_{i=1}^{N} o_{i}<p_{i} \\
& \mathbf{o} \geq \mathbf{p} \text { if } \forall_{i=1}^{N} o_{i} \geq p_{i} \\
& \mathbf{o}>\mathbf{p} \text { if } \forall_{i=1}^{N} o_{i}>p_{i} .
\end{aligned}
$$

One can regard the histogram as a single measurement of a $M$-dimensional random vector $\boldsymbol{b}$ with a realization $\mathbf{b}$ for which the joint pdf is given by a multinomial distribution, as shown in G. Cowan (1998)

$$
p_{\boldsymbol{b}}(\mathbf{b} ; \epsilon(\boldsymbol{\theta}))=\frac{N !}{b_{1} ! \ldots b_{M} !}\left(\frac{\epsilon_{1}(\boldsymbol{\theta})}{N}\right)^{b_{1}} \ldots\left(\frac{\epsilon_{M}(\boldsymbol{\theta})}{N}\right)^{b_{M}} .
$$

Thus, the probability to be in bin $m$ is expressed as the expectation $\epsilon_{m}(\theta)$ divided by the number of observations $N$. The loglikelihood is given by

$$
l(\boldsymbol{\theta})=\log (L(\mathbf{b} ; \epsilon(\boldsymbol{\theta})))=\log \left(p_{\boldsymbol{b}}(\mathbf{b} ; \epsilon(\boldsymbol{\theta}))\right) .
$$

Dropping all terms that are not dependent on $\boldsymbol{\theta}$ we can write

$$
l(\boldsymbol{\theta})=\arg \max _{\boldsymbol{\theta}} \sum_{m=1}^{M} b_{m} \log \left(\epsilon_{m}(\boldsymbol{\theta})\right) .
$$

In our case, this can be solved by a grid-search in $\boldsymbol{\theta}$ based on (2) using an appropriate model for $s_{I}(t ; \boldsymbol{\theta})$ and $s_{Q}(t ; \boldsymbol{\theta})$ following (5) and (6) to derive $\epsilon_{m}(\boldsymbol{\theta})=N p_{m}(\boldsymbol{\theta})$. Additionally, the receive antenna gain $G_{r} \approx 50 \mathrm{~dB}$ and the beamwidth of the antenna is so small that the signal of only one GNSS satellite is received with a signal-to-noise-ratio (SNR) of approximately 25-30 dB. Thus, the histogram of $\mathbf{x}[n]$ with bin limits $\mathbf{x}_{m}^{\min }$ and $\mathbf{x}_{m}^{\max }$ resembles the so-called signal modulation constellation diagram. Usually the signal modulation constellation diagram is depicted in a two-dimensional graph with $x_{I}[n]$ on the horizontal axis and $x_{Q}[n]$ on the vertical axis and a color scale showing the absolute frequency of entries in the bins.

In the next section, we will assess the performance of the proposed estimator given in (19) using high gain antenna measurement data.

\section{ESTIMATION OF SLANT TEC AND BROADBAND IONOSPHERIC CORRECTION}

We propose that the characteristic distortion of the signal modulation constellation diagram is exploited to estimate the ionospheric TEC as described in (9) - (19), especially in the case of high gain antenna measurements (Thoelert et al. 2013). Figure 2 illustrates the working principle of the maximum likelihood TEC estimator as described in (19).

The graph on the left-hand side shows the constellation histogram of a measured and Doppler corrected Galileo E5 signal. A characteristic like a "buzz saw blade" can be observed. And indeed, if the measured signal is corrected with the dispersive ionosphere model at 82 TECU the signal power is shifted remarkably close to the nominal constellations shown in the right part of Figure 2. We attribute the remaining distortion to the satellite payload, the antenna phase, and noise. Nevertheless, the striking improvement of the signal shape is a strong indicator that the TEC was correctly estimated.

We have also analyzed a complete satellite path with data acquisitions every 5 minutes and performed slant TEC estimations using each snapshot. Figure 3 shows the result of the TEC estimation based on the Doppler corrected I-Q-samples and based on the method described within this paper (solid red line). In comparison, the TEC values calculated based on a vertical TEC map, are presented. For this processing, the simple thin-shell ionospheric layer model has been used to convert the vertical TEC map information into slant TEC values corresponding to the signal propagation path. 


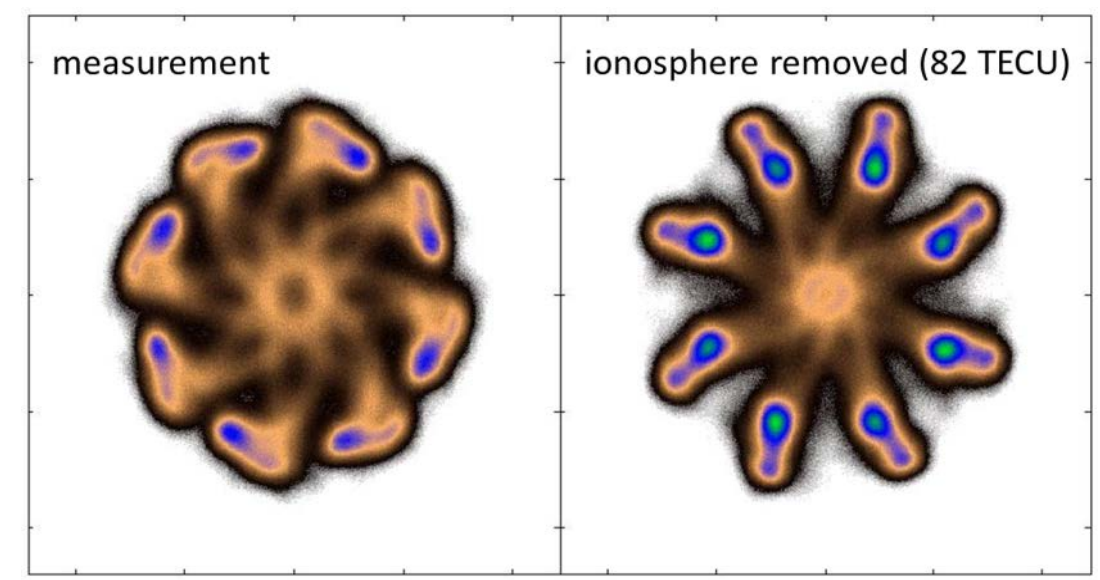

Fig. 2 Constellation histogram of Doppler corrected Galileo E5 measurement data without (left) and with an ionospheric correction of estimated 82 TECU applied (right).

Furthermore, a GNSS receiver (Septentrio PolaRx4TR) was connected in parallel to the high gain antenna during the I-Qsample recording. Dual-frequency GNSS observables can be used to calculate the slant TEC values. The challenge in this approach is the correction of the additional biases based on the satellite payload and GNSS receiver behavior. The first one can be compensated using IGS MGEX products. Using the provided differential code bias (DCB) of the observed satellite, a TEC map, and measurement observables, one can calculate the DCB of the used receiver. For this procedure, the discrepancy between the TEC map information and the actual ionospheric conditions during the calibration has to be negligible. To achieve this, receiver observables at high elevation during a nighttime satellite pass were utilized, when the vertical and slant TEC are nearly identical and the absolute influence of the ionosphere is minimal. Now one can determine the slant TEC based on the GNSS dual-frequency measurements. These results are shown in the dashed curve (green) in Figure 3.

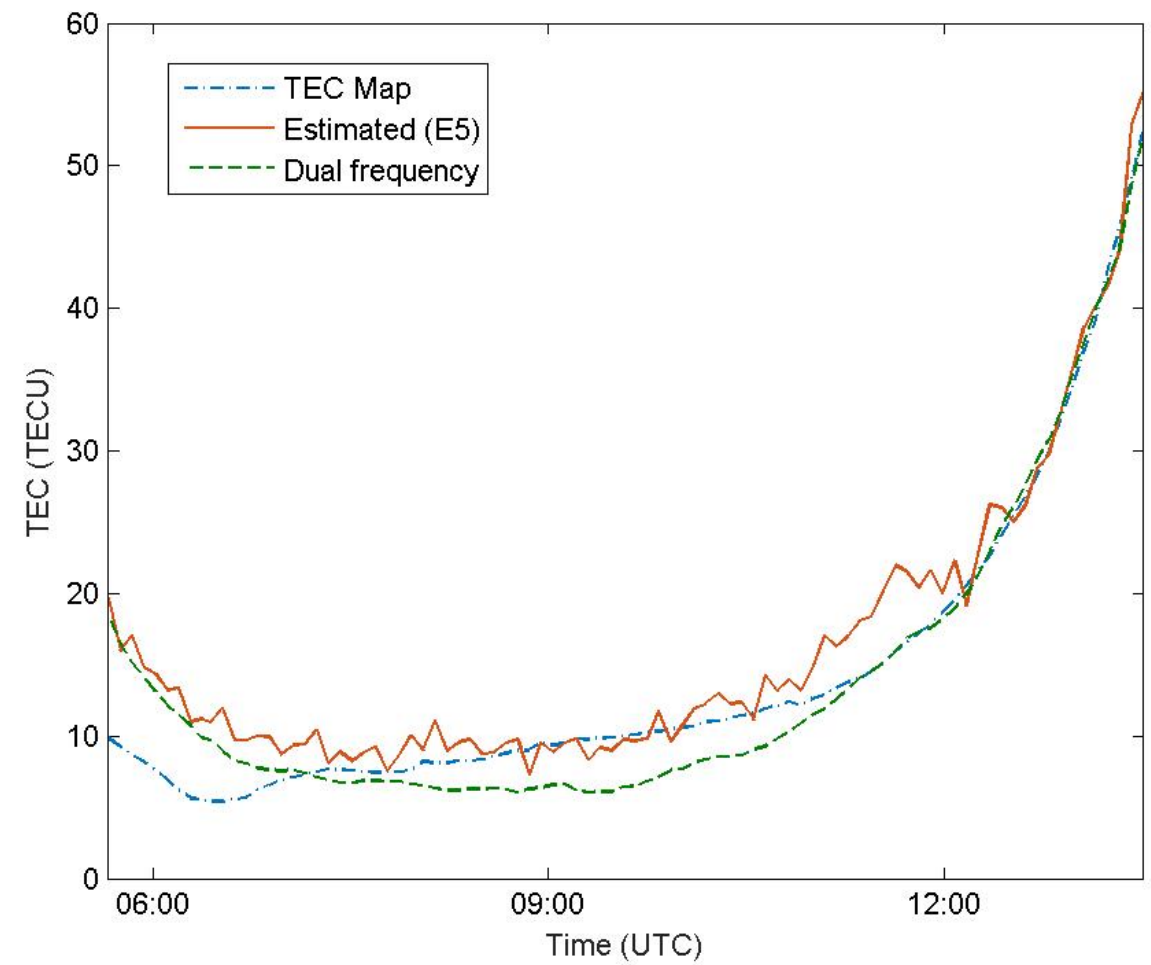

Fig. 3 Slant TEC values regarding a satellite path observed from Weilheim ground station, Germany. The dash-dotted curve (blue) represents TEC values calculated on the basis of a vertical TEC Map and the use of the thin-shell ionospheric layer model. The dashed curve (green) shows TEC estimates based on receiver measurements recorded at the same station. The solid curve (red) represents the estimated TEC values based on the Doppler corrected I and Q samples and the method described above. 
The mean and standard deviation of the relation of ionospheric TEC estimation based on the I-Q-samples regarding the TEC map and the GNSS receiver results are given in Table 1, respectively.

Table 1: Mean and standard deviation of ionospheric TEC estimation based on the I-Q-samples related to the TEC map and GNSS receiver results, respectively.

\begin{tabular}{|l|l|l|}
\hline $\begin{array}{l}\text { lonospheric TEC estimates based on I-Q- } \\
\text { samples compared to: }\end{array}$ & Mean difference & Standard deviation \\
\hline Dual frequency & 2.4 TECU & 1.6 TECU \\
\hline TEC map & 2.1 TECU & 2.5 TECU \\
\hline
\end{tabular}

The residuals show good performance of our proposed method in comparison to the other two common ionospheric TEC estimation methods, using TEC maps or a GNSS receiver connected to the high gain antenna. A literature review provides an accuracy between 2 and 9 TECU according to IGS TEC map products (Johnston 2017) and around 1 TECU (RMS) for very precise TEC map products (Jakowski 2017). But note that also between TEC map products a difference up to 12 TECU can be present (Li 2017). For low TEC (low TECU), the results are by trend a bit higher than from the two other methods. For higher TEC (high TECU), the results seem to be a bit more stable (see Figure 3 for TEC higher than 20 TECU).

\section{CONCLUSION}

In this work, we have proposed a novel method to estimate the ionospheric electron content directly from the single frequency signal high gain antenna measurements. We have demonstrated that the proposed method to estimate slant TEC based on I-Qsamples from a high gain antenna performs at least similarly well as methods based on TEC maps or using a calibrated GNSS receiver connected to the high gain antenna (dual-frequency measurements). We would like to point out that high gain antenna measurements are independent of code correlation. Additionally, the received signal is de facto unaffected by multipath and interference because of the narrow aperture angle $\left(0.5^{\circ}\right)$ of the antenna. Our proposed method thus is inherently free of these uncertainties that might otherwise be critical in the case of conventional GNSS receiver measurements.

Caused by the used data basis the proposed method could not be used in typical mass-market GNSS receivers, but the method can provide independent ionosphere TEC value estimation for approval purposes of the receiver based TEC map estimation. Further developments in receiver architecture regarding the use in signal performance monitoring systems are going in the direction of multi-correlator receivers, which would also be a common application for wideband single frequency TEC estimation and correction. Additionally, the application could be very useful during in-orbit tests of new satellites. At this time no direct dual frequency analysis for TEC estimation is available because mostly these types of satellites are set unhealthy until the complete evaluation of their signal quality.

\section{ACKNOWLEDGMENTS}

The authors want to thank the colleagues from the German Space Operation Center (GSOC), at DLR's ground station Weilheim, for supporting and operating the high gain antenna. Furthermore, the authors thank our colleagues Volker Wilken, Martin Kriegel, Jens Berdermann and Mainul Hoque from DLR Neustrelitz, for the provision of high-resolution TEC maps.

\section{REFERENCES}

\section{G. Cowan (1998) Statistical Data Analysis. Clarendon Press, Oxford}

Gao GX, Datta-Barua S, Walter T, Enge P (2007) Ionosphere effects for wideband GNSS signals. Proc. of the 63rd Annual Meeting of the Institute of Navigation, April 23 - 25, Cambridge, MA, USA, 147-155

Henkel P, Gao GX, Walter T, Günther C (2009) Robust Multi-Carrier, Multi-Satellite Vector Phase Locked Loop with Wideband Ionospheric Correction and Integrated Weighted RAIM. Proc. ENC GNSS 2009, Naples, Italy

T. Hobinger and N. Jakowski (2017), Atmospheric signal propagation. Springer Handbook of Global Navigation Satellite Systems, P. J. Teunissen and O. Montenbruck, Eds. Springer International Publishing, 2017, 165-194 
Jakowski N, Mayer C, Hoque MM, Wilken V (2011) Total electron content models and their use in ionosphere monitoring. Radio Science, 46(6):RS0D18

Kelley MC (2009) Earth’s Ionosphere: Plasma Physics \& Electrodynamics. Elsevier Science, Burlington Klobuchar JA (1987) Ionospheric Time-Delay Algorithm for Single-Frequency GPS Users. IEEE Transactions on Aerospace and Electronic Systems, vol. AES-23(3):325-331, doi: 10.1109/TAES.1987.310829

Thoelert S, Furthner J, Meurer M (2013) GNSS Survey - Signal Quality Assessment of the latest GNSS satellites. Proc. ION ITM 2013, Institute of Navigation, January 28-30, San Diego, USA, 608 - 615

Ya’acob N, Abdullah M, Ismail M (2008) Determination of GPS Total Electron Content using Single Layer Model (SLM) Ionospheric Mapping Function. International Journal of Computer Science and Network Security, 8(9):154-160

Zolesi B, Cander LR (2014) Ionospheric Prediction and Forecasting. Springer Berlin Heidelberg

Johnston G., Riddell A., Hausler G. (2017), The International GNSS Service. Springer Handbook of Global Navigation Satellite Systems, P. J. Teunissen and O. Montenbruck, Eds. Springer International Publishing, p. 975, Table 33.6

Jakowski N. (2017), Ionosphere Monitoring. Springer Handbook of Global Navigation Satellite Systems, P. J. Teunissen and O. Montenbruck, Eds. Springer International Publishing, p. 1143, Fig. 39.5

Li, M., Yuan, Y., Wang, N. et al. J Geod (2017) 91: 279. https://doi.org/10.1007/s00190-016-0962-1 